\title{
Psoriatic Spondylitis
}

Spondylitis in Active and Inactive Phases

\author{
Spondylodiscitis
}

- A 65-year-old male

- Chronic dorsal and lumbar pain

- Stiffness

- Presence of HLA-B27
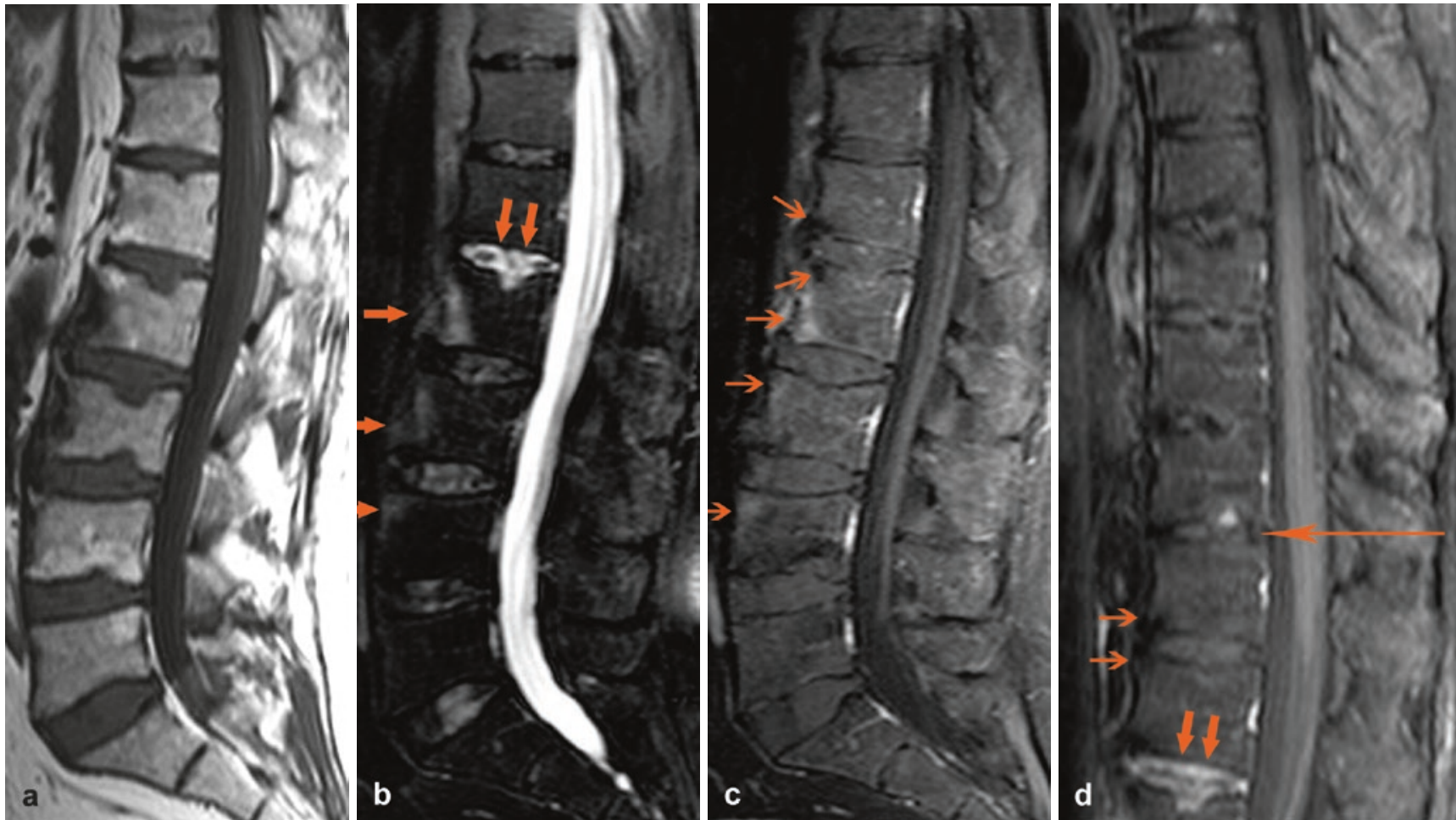

Fig. 1 Sagittal SE T1-weighted image (a), sagittal TSE T2-weighted image with fat saturation (b), sagittal SE T1-weighted images with fat saturation after the administration of contrast medium (c, d). In this patient, we can find multiple areas of spondylitis in different phases. Active anterior spondylitis at L2/L3 and L3/L4, with edema and contrast enhancement in the anterior edges of the vertebral bodies $(\mathbf{a}-\mathbf{c})$. In

addition, a florid Andersson lesion (spondylodiscitis) is seen at T11/ T12, with a small area of contrast enhancement (d). Syndesmophyte at L1/L2 (a). Chronic phase of anterior spondylitis in dorsal spine, with no contrast enhancement of the anterior edges of the vertebral bodies (double thin arrows, $\mathbf{d}$ ) 